\title{
Mitochondrial transfer from mesenchymal stem cells to neural stem cells protects against the neurotoxic effects of cisplatin
}

\author{
Nabila Boukelmoune, Gabriel S. Chiu, Annemieke Kavelaars and Cobi J. Heijnen * (D)
}

\begin{abstract}
Mesenchymal stem cells (MSCs) transfer healthy mitochondria to damaged acceptor cells via actin-based intercellular structures. In this study, we tested the hypothesis that MSCs transfer mitochondria to neural stem cells (NSCs) to protect NSCs against the neurotoxic effects of cisplatin treatment. Our results show that MSCs donate mitochondria to NSCS damaged in vitro by cisplatin. Transfer of healthy MSC-derived mitochondria decreases cisplatin-induced NSC death. Moreover, mitochondrial transfer from MSCs to NSCs reverses the cisplatin-induced decrease in mitochondrial membrane potential. Blocking the formation of actin-based intercellular structures inhibited the transfer of mitochondria to NSCs and abrogated the positive effects of MSCs on NSC survival. Conversely, overexpression of the mitochondrial motor protein Rho-GTPase 1 (Miro1) in MSCs increased mitochondrial transfer and further improved survival of cisplatin-treated NSCS.

In vivo, MSC administration prevented the loss of DCX+ neural progenitor cells in the subventricular zone and hippocampal dentate gyrus which occurs as a result of cisplatin treatment. We propose mitochondrial transfer as one of the mechanisms via which MSCs exert their therapeutic regenerative effects after cisplatin treatment.
\end{abstract}

Keywords: Mesenchymal stem cells, Neuronal stem cells, Mitochondrial transfer, Cisplatin

\section{Introduction}

Mesenchymal stem cells (MSCs) have been shown to stimulate tissue repair in various disease models, including cardiomyopathy, pulmonary damage, cerebral ischemic insults, and neurodegenerative disorders like Alzheimer and Parkinson disease [8]. Multiple mechanisms have been proposed to mediate these beneficial effects of MSCs, including suppression of inflammation and release of growth factors. Recently, it has become apparent that transfer of healthy mitochondria to damaged cells represents an important mechanism of endogenous regeneration. For example, astrocytes have been shown to transfer mitochondria to neurons after ischemic stroke in mice [15]. MSCs also transfer mitochondria to cardiomyocytes in a model of anthracycline-induced cardiomyopathy [46], to murine alveoli in acute lung injury [17], to murine lung epithelial cells in rotenone-induced airway injury [2], to cortical neurons in a model of cerebral stroke [5]

\footnotetext{
* Correspondence: cjheijnen@mdanderson.org

Laboratory of Neuroimmunology, Department of Symptom Research, The
University of Texas MD Anderson Cancer Center, 1515 Holcombe Blvd, Unit

Laboratory of Neuroimmunology, Department of Symptom Research, The 384, Houston, TX 77030, USA
}

and to human monocyte-derived macrophages as well as murine alveolar macrophages in models of acute respiratory distress syndrome [18].

Platinum-based chemotherapeutic agents, such as cisplatin, are widely used to treat solid tumors [19]. Increasing evidence indicates that cognitive deficits develop in cancer patients treated with chemotherapy. Cognitive deficits induced by cancer treatment are characterized by confusion, memory loss, reduced attention and processing speed, and decreased executive functioning [20-22, 39, 43]. Longitudinal neuropsychological studies report that up to $75 \%$ of cancer patients experience cognitive problems during treatment and likely $35 \%$ of affected cancer patients have long-term cognitive effects that seriously impair their quality of life [1].

We recently reported that cisplatin induces cognitive impairment, synaptosomal mitochondrial dysfunction, and changes in neuronal mitochondrial morphology in mice [7]. Nasal administration of MSCs to cisplatin-treated mice restored cognitive function and normalized mitochondrial function [6]. Lomeli et al. have demonstrated that 
MSCs reduce cranial irradiation-induced brain damage as well [28].

Neurogenic precursor proliferation and differentiation, particularly in the dentate gyrus (DG) of the hippocampus, is crucial to formation of new neurons, thereby enhancing neural circuity and improving learning and memory [37, 41, 47]. Disruption of adult neurogenesis contributes to the pathogenesis of many neurodegenerative diseases associated with cognitive impairment. In vivo and in vitro, cisplatin induces loss of neuronal precursors [3, 7, 28]. Gong el al. have shown that neuronal precursors are very sensitive to low cisplatin concentrations [13].

The aim of the present study was to test the hypothesis that MSCs can protect neuronal stem cells (NSCs) in culture, in vitro against the neurotoxic effects of cisplatin through mitochondrial donation. Moreover, we investigated whether nasal administration of MSCs in vivo protects the brain neurogenic pools against the damaging effects of cisplatin.

\section{Materials and methods Animals}

9 weeks old C57BL/6 J male mice (Jackson Laboratory) were used. Mice were housed on a $12 / 12 \mathrm{~h}$ reverse dark-light cycle. Animals had access to water and food ad libitum. All experiments were conducted at The University of Texas MD Anderson Cancer Center in Houston, Texas. Animals were used in accordance with Institutional Animal Care and Use Committee-approved protocols.

\section{Cell culture and transfection}

Mouse cortical NSCs (R\&D Systems, Minneapolis, $\mathrm{MN}$, USA) were cultured in monolayers in low-glucose Dulbecco's Modified Eagle's Medium (DMEM)/F12, supplemented with $100 \mathrm{U} / \mathrm{mL}$ penicillin and N-2 plus supplement (R\&D Systems). Fibroblast growth factor basic and epidermal growth factor (both $20 \mathrm{ng} / \mathrm{mL}$; R\&D Systems) were added to the cultures daily. NSCs were cultured on surfaces coated with poly-L-ornithine (Sigma-Aldrich, St. Louis, MO, USA) and bovine fibronectin (R\&D Systems) and detached using Accutase (Innovative Cell Technologies, San Diego, CA, USA).

C57BL/6 mouse MSCs (Invitrogen) were grown in 5\% $\mathrm{CO}_{2}$ at $37^{\circ} \mathrm{C}$ in DMEM/F12 medium with GlutaMax-I, supplemented with $10 \%$ MSC-qualified fetal bovine serum and $5 \mu \mathrm{g} / \mathrm{mL}$ gentamycin (all from GIBCO, Carlsbad, CA, USA). Before transfection, MSCs were seeded on plates coated with poly-L-lysine (Sigma-Aldrich). Cells were harvested using TrypLE-express (GIBCO). For labeling mitochondria we used pLYS1-FLAG-MitoGFP-HA (Addgene plasmid \# 50057; gift from Vamsi Mootha [35]) which contains the pore-forming subunit of the mitochondrial calcium uniporter to target the label to the mitochondria. The mito-mcherry plasmid was generated by cloning the mito-GFP insert of the pLYS1-FLAG-MitoGFP-HA plasmid into the mcherry2-N1 vector (Addgene plasmid \# 54517, gift from Michael Davidson). The mitochondrial rho GTPase 1 (Miro1)-GFP overexpression plasmid (RhoT1, MG224107) was obtained from Origene (Rockville, MD, USA). Transfection of mito-GFP, mito-mcherry and Miro1-GFP plasmids were performed using either Lipofectamine LTX (Invitrogen) with Plus reagent for mito-GFP or mito-mcherry expression in MSCs or Lipofectamine 2000 (Invitrogen) and JetPrime (Polyplus Transfection, New York, NY, USA) for Miro1 overexpression, according to the manufacturer's instructions.

\section{Chemotherapy and MSC treatment}

Cisplatin $(2.3 \mathrm{mg} / \mathrm{kg} /$ day; Teva, Petah Tiva, Israel) or phosphate-buffered saline (PBS) was administered daily to mice intraperitoneally, following a 5 days injections and 5 days rest scheme. Prior to MSC application, mice received $3 \mu \mathrm{l}$ of hyaluronidase in PBS in each nostril (100 U per mouse, Sigma-Aldrich) to increase the permeability of the nasal mucosa $[6,10,11,16] .30 \mathrm{~min}$ after, $3 \mu \mathrm{l}$ of MSC cell suspension $\left(1 \times 10^{6}\right.$ cells per mouse per day) or PBS were administered to mice, twice in each nostril, for a total of $12 \mu \mathrm{l}$. MSCs were applied 48 and $96 \mathrm{~h}$ after the last cisplatin injection.

\section{Co-culture of MSCs and NSCs}

NSCs $\left(35 \times 10^{4}\right.$ cells) were plated on cell culture imaging dishes (ibidi, Fitchburg, WI, USA). Two days after plating, NSCs were treated with cisplatin (Teva, Petah Tikva, Israel) for $8 \mathrm{~h}$, stained with $20 \mu \mathrm{M}$ CellTracker Blue fluorescent probe (CTB; Invitrogen, Carlsbad, CA) for $45 \mathrm{~min}$ at $37^{\circ} \mathrm{C}$, and washed in serum-free media. MSCs $\left(15 \times 10^{4}\right.$ cells) were added to the culture for $17 \mathrm{~h}$. Co-cultures were stained with wheat germ agglutinin (WGA) conjugates (WGA 488 or WGA 594, 1/300 dilution; Invitrogen) for $10 \mathrm{~min}$ at $37^{\circ} \mathrm{C}$, followed by 2 washes in Hank's Balanced Salt Solution (GIBCO) prior to imaging in Live Cell Imaging Solution (Invitrogen).

To inhibit actin polymerization, MSCs were treated with $2 \mu \mathrm{M}$ Latrunculin B (Sigma-Aldrich) for $24 \mathrm{~h}$ prior to co-culture with NSCs. To assess NSC survival, CTBpositive NSCs were counted using the countess II FL automated cell counter (Invitrogen). Mitochondrial transfer was quantified in representative confocal images of every condition used, and the percentage of either mito-GFP-positive or mito-mcherry-positive NSCs was determined. 


\section{Analysis of mitochondrial membrane potential}

After exposure to cisplatin and co-culture with MSCs, cells were stained with $250 \mathrm{nM}$ of the fluorescent mitochondrial membrane potential-sensitive dye tetramethylrhodamine methyl ester (TMRM, Invitrogen) for $45 \mathrm{~min}$ at $37^{\circ} \mathrm{C}$. As a positive control, NSCs were treated with $10 \mu \mathrm{M}$ carbonilcyanide p-triflouromethoxyphenylhydrazone (FCCP), a mitochondrial uncoupler, for $15 \mathrm{~min}$. Cells were imaged by confocal microscopy or washed with serum-free media and collected in a single cell suspension for flow cytometry analysis. The fluorescence signal was detected with a BD accuri C6 Flow Cytometer (BD Biosciences, San Jose, CA USA) at FL2 emission of $585 / 40 \mathrm{~nm}$. We used the TMRM in sub-quench mode, as described previously [30,33].

\section{Analysis of mitochondrial bioenergetics}

To assess mitochondrial bioenergetics, NSCs were grown in a Seahorse XFe 24 microplate (Seahorse Biosciences/ Agilent Technologies, Santa Clara, CA, USA) coated with Poly-L-Ornithine and fibronectin to $80 \%$ confluency. NSCs were treated with $0.5-1 \mu \mathrm{M}$ cisplatin or vehicle for $12 \mathrm{~h}$, washed with serum-free media, and incubated for 1 $\mathrm{h}$ at $37^{\circ} \mathrm{C}$ in XF base media (Seahorse Biosciences) supplemented with $11 \mathrm{mM}$ glucose, $2 \mathrm{mM}$ glutamine, and 1 $\mathrm{mM}$ pyruvate. Oligomycin $(2 \mathrm{mM})$, FCCP $(4 \mathrm{mM})$, and rotenone/antimycin A (2 $\mathrm{mM}$ each) were used with a 3-time repeat of a 2-min mix, 3-min wait, and 2-min measure assay cycle. Oxygen consumption rates were normalized to the total protein content of each well. Basal respiration, adenosine triphosphate (ATP)-linked respiration, proton leak, and maximal respiratory capacity were determined as described previously $[7,23]$.

\section{Immunohistochemistry}

Immunostaining of neural precursors in the dentate gyrus (DG) and subventricular zone (SVZ) was performed as previously reported [7]. Briefly, mice were euthanized and perfused with PBS followed by $4 \%$ paraformaldehyde (PFA). Brains were removed and fixed in 4\% PFA for $48 \mathrm{~h}$, paraffin embedded and sectioned at 8 and $10 \mu \mathrm{m}$ for the SVZ and DG, respectively. Brain slices were stained for doublecortin (DCX, 1:50; Abcam, Cambridge, UK) followed by AlexaFluor 488 Donkey secondary antibody (Invitrogen) and DAPI. Sections were imaged and positive staining was quantified using ImageJ. The number of DCX+ cells was quantified in representative images by researchers blinded to treatment.

To characterize NSCs, immunostaining was done as previously described [4]. Briefly, cells were fixed with $4 \%$ paraformaldehyde in PBS, treated with $0.25 \%$ Triton $\mathrm{X}-100$, blocked in $2 \%$ BSA in PBS and stained with either anti-DCX antibody (1:50; Abcam, Cambridge, UK), anti Sox2 (1/200; Millipore, Burlington, MA), anti BIII-Tubulin (1/200; R\&D Systems) or anti Nestin
(1/1000, Abcam) and anti-GFAP (1/200, Acris, Rockville, MD) in blocking buffer followed by secondary antibody and DAPI. NSCs used for experiments were $52 \%$ Nestin +, 17\% GFAP +, 16\% Nestin/GFAP double-positive, 92\% DCX+, 94\% Sox $2+$ and 5\% BIII-Tubulin+.

\section{Confocal microscopy}

Live cell images of MSC and NSC co-cultures, fixed NSC staining as well as DCX expression in the DG and SVZ, were acquired using a SPE Leica Confocal Microscope (Leica Microsystems, Buffalo Grove, IL, USA) with a $63 \mathrm{X}$ or $40 \mathrm{X}$ objective, and analyzed with LAS X software. Image $J$ was used to quantify TMRM intensity in individual cells. Data are expressed as the corrected total cell fluorescence $=$ integrated density $-($ area of selected cell $\times$ mean fluorescence of background readings) [31].

\section{Statistical analysis}

Data are presented as mean \pm standard error of the mean (SEM) of at least 3 independent experiments. Data were analyzed using GraphPad Prism 7 (GraphPad Software, La Jolla, CA, USA). One-way or two-way analysis of variance (ANOVA) was used with or without repeated measure followed by either Bonferroni's or Tukey's correction for multiple comparisons, Dunn's multiple comparison or using Student's t-test, as appropriate and indicated in the legends.

\section{Results}

MSCs rescue NSC from cisplatin-induced cell death in vitro and in vivo

NSCs were treated with $0,0.5$ or $1 \mu \mathrm{M}$ cisplatin for $8 \mathrm{~h}$ and stained with Cell Tracker Blue fluorescent probe. Subsequently, the Cell Tracker Blue positive neurons were co-cultured with MSCs for $17 \mathrm{~h}$ and recovery of the NSCs was quantified. Figure 1a shows that cisplatin dose-dependently reduced NSC survival. Addition of MSCs significantly increased survival of NSCs.

Next, we tested whether MSCs also rescue NSCs from cisplatin-induced cell loss in the DG of the hippocampus and the SVZ. Mice were injected with cisplatin during two cycles of 5 days $(2.3 \mathrm{mg} / \mathrm{kg})$ with 5 days of rest in between $[7,23]$. One month after completion of cisplatin treatment, we observed a $40 \%$ decrease in the DCX+ neural progenitors in the DG of the hippocampus (Figs. 1f) and a $50 \%$ decrease in the SVZ (Fig. 1k). Nasal application of MSCs at 48 and $96 \mathrm{~h}$ after the last cisplatin dose reduced the cisplatin-induced loss of DCX+ neuronal progenitors in both the DG (Figs. 1f) and SVZ (Figs. 1k).

\section{Cisplatin induces mitochondrial damage in NSCs}

To assess whether cisplatin induced mitochondrial damage in NSCs, we measured oxygen consumption rates of NSC using the Seahorse XF24 extracellular flux analyzer (Fig. 2a). NSCs treated with cisplatin showed a marked 

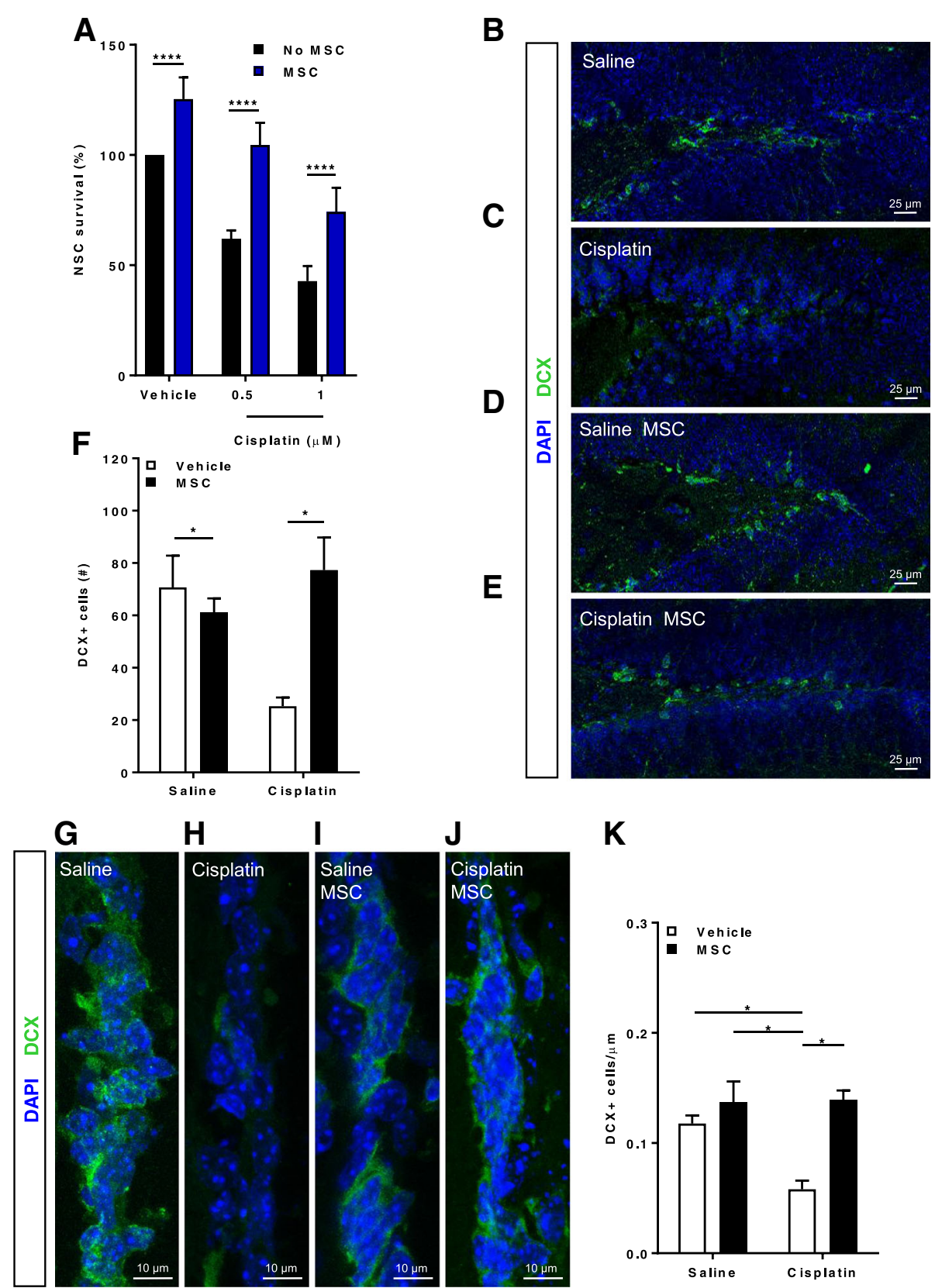

K

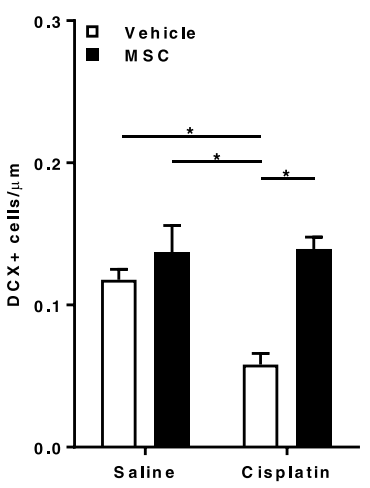

Fig. 1 MSCs rescue damaged NSCs after cisplatin treatment and reverse the loss of neuroblasts in the brain. a Neuronal stem cells (NSCs) were treated with cisplatin or vehicle for $8 \mathrm{~h}$, stained with cell tracker blue (CTB), and subsequently co-cultured for $17 \mathrm{~h}$ with or without mesenchymal stem cells (MSCs). Survival of NSCS was assessed by counting the number of CTB-positive cells. The graph shows the rate of NSC survival after $17 \mathrm{~h}$ co-culture with MSCS (blue bars) or without MSCs (black bars). Data are normalized to survival in the absence of MSCs and cisplatin in each experiment and represent the mean \pm SEM of 6 independent experiments. Data were analyzed using two-way ANOVA, repeated measures (cisplatin $\times$ MSC interaction: $P<0.01$ ), followed by Bonferroni's post-hoc test. ${ }^{* * *} P<0.0001$ ). (b-k) Animals were treated with cisplatin for 2 cycles of 5 days. DCX+ neuronal progenitors were observed in the DG of the hippocampus (b-e) as well as the SVZ ( $\mathbf{g}-\mathbf{j})$. The number of cells were counted in the DG tip (f). For the SVZ, the number of cells was normalized to the length of the SVZ (k). Data were analyzed by two-way ANOVA followed by Tukey's post-hoc test. ${ }^{*} P<0.05$

decrease in basal respiration as well as in oxygen consumption related to ATP production in comparison to control conditions. Furthermore, cisplatin significantly reduced maximal respiration as measured in the presence of FCCP (Fig. 2b).
As a second measure of cisplatin-induced loss of mitochondrial integrity, we assessed mitochondrial membrane potential. NSCs were treated with cisplatin and labeled with the mitochondrial membrane potential-sensitive dye tetramethylrhodamine methyl ester (TMRM). Using live-cell 

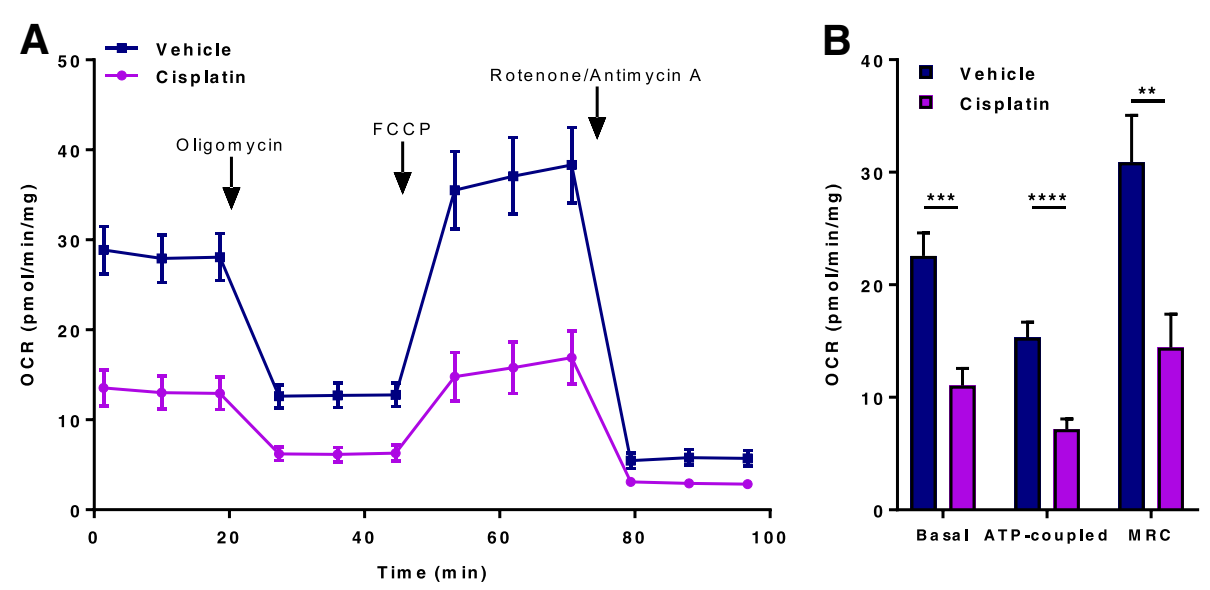

C
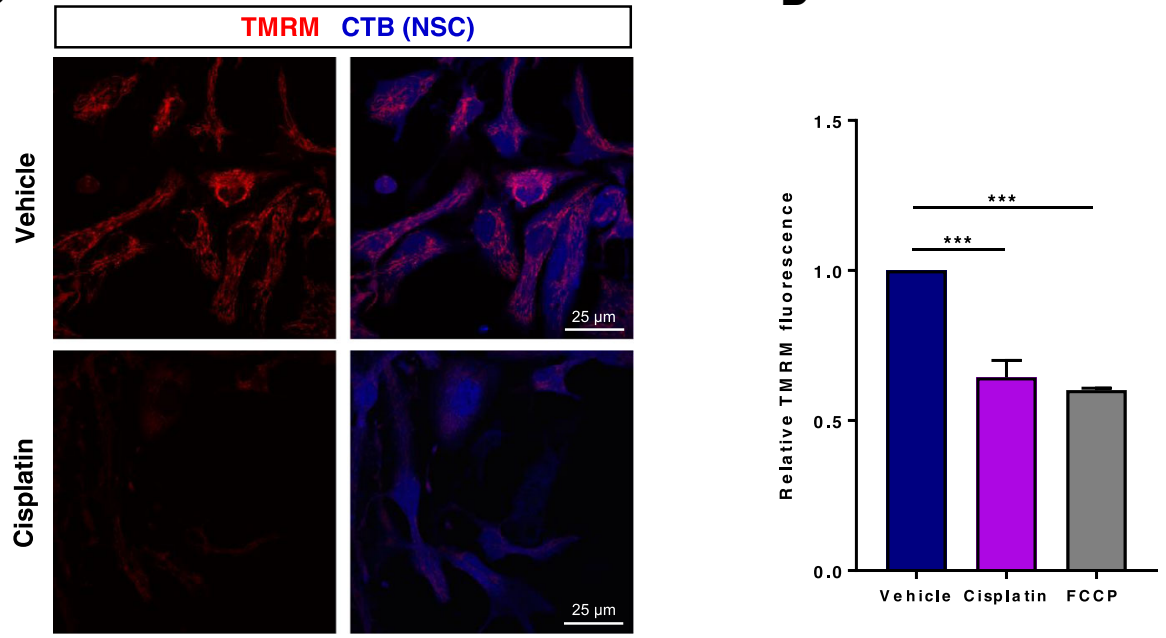

Fig. 2 Cisplatin induces NSC mitochondrial dysfunction. Neuronal stem cells (NSCs) were treated with $1 \mu \mathrm{M}$ cisplatin for $12 \mathrm{~h}$. Oxygen consumption rates (OCR) were analyzed using a Seahorse XFe 24 Analyzer and normalized to protein content (a). Mean basal, ATP production-related, and maximum respiratory capacity (MRC) normalized to protein content were calculated (b). Results are expressed as means \pm SEM of 3 independent experiments. Data were analyzed using Student's t-test: ${ }^{* *} P<0.01 ;{ }^{* *} P<0.001 ; * * * P<0.0001$. NSCs were treated with $1 \mu \mathrm{M}$ cisplatin for $8 \mathrm{~h}$ followed by $17 \mathrm{~h}$ in normal medium. Cells were stained with tetramethylrhodamine methyl ester (TMRM) and monitored immediately by live-cell imaging (c) or flow cytometry (d). NSCs treated with carbonilcyanide p-triflouromethoxyphenylhydrazone (FCCP, $10 \mu \mathrm{M}$ for $15 \mathrm{~min}$ ) were used as a positive control. Bar graphs represent mean \pm SEM of 3 independent experiments. Data are normalized to mean fluorescence intensity of vehicle-treated cells in each experiment. Data were analyzed using One-way ANOVA followed by Bonferroni's post-hoc test. ${ }^{* * *} P \leq 0.001$

imaging, we observed that cisplatin decreased the TMRM signal, indicating a reduction in mitochondrial membrane potential (Fig. 2c). Quantification of the change in TMRM staining by flow cytometry showed that exposure of NSCs to cisplatin decreased mean TMRM fluorescence intensity by approximately $40 \%$. This decrease in mitochondrial membrane potential was similar to what we observed in response to the mitochondrial uncoupler FCCP, which was used as a positive control (Fig. 2d).

\section{Mitochondrial transfer from MSC to cisplatin-treated NSC} Having shown that MSCs can protect NSCs against cisplatin-induced cell death, we hypothesized that the mechanism of the restorative effect of MSCs may involve donation of healthy mitochondria from MSCs to cisplatin-damaged NSCs. To test this possibility, we labeled the mitochondria in the MSCs using mito-GFP (Figs. 3) or mito-mcherry (Fig. 4) plasmids [35]. NSCs were treated with cisplatin or control medium for $8 \mathrm{~h}$, followed by co-culture with MSCs containing the fluorescently tagged mitochondria. Confocal imaging revealed GFP+ or mcherry+ mitochondria in cisplatintreated NSCs, suggesting mitochondrial transfer from MSCs to NSCs (Figs. 3 and 4). Quantitative assessment of mitochondrial transfer showed that cisplatin dose-dependently increased the percentage of NSC that had received mitochondria from MSCs (Fig. 3c). The orthogonal slice view in Fig. 4c demonstrates that the MSC-derived mitochondria are indeed localized inside the cisplatin-treated NSC. 

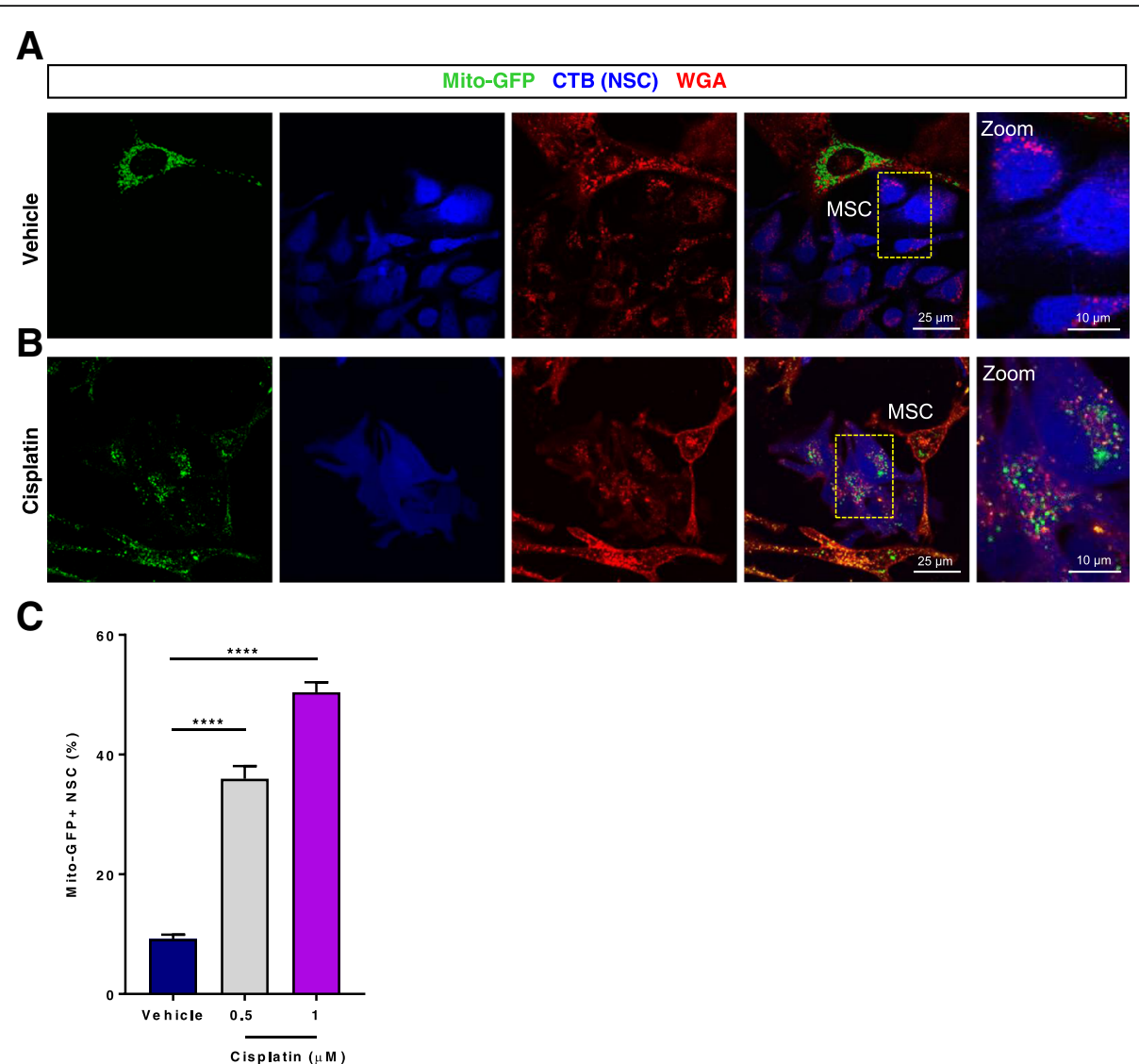

Fig. 3 MSCs donate mitochondria to NSCs damaged by cisplatin. Representative confocal images of Neuronal stem cells (NSCs) treated with $1 \mu$ M cisplatin or vehicle for $8 \mathrm{~h}$, stained with cell tracker blue (CTB) and subsequently co-cultured for $17 \mathrm{~h}$ with mesenchymal stem cells (MSC) transfected with mito-GFP (a and b) to label the MSC-derived mitochondria. Prior to confocal imaging, co-cultures were stained with wheat germ agglutinin (WGA) AF 594 (a and b) to reveal cell membranes. GFP-positive mitochondria were detected in NSCs treated with cisplatin (b, right panel), indicating mitochondrial transfer. The extent of mitochondrial transfer was quantified by counting the number of NSCs positive for the GFP signal in the co-cultures (c). Data are represented as the mean \pm SEM of 3 independent experiments and were analyzed using One-way ANOVA followed by Bonferroni's post hoc-test. ${ }^{* * * *} P<0.0001$

MSC normalize mitochondrial membrane potential in cisplatin-treated NSCs

Cisplatin treatment decreased TMRM staining in NSCs, indicating a reduction in mitochondrial membrane potential (Figs. 2 and 5). We next analyzed whether mitochondrial transfer of MSCs to cisplatin-treated NSCs restored their mitochondrial membrane potential. To that end, we compared TMRM staining in cisplatin-treated NSCs co-cultured with or without mito-GFP-transfected MSCs (Fig. 5). TMRM staining was markedly increased in those NSCs that received mitochondria from the MSCs (as shown by the presence of mito-GFP-labeled mitochondria) in comparison with the NSCs that were negative for mito-GFP (Fig. 5). The latter results indicate that MSC-derived mitochondrial donation restores mitochondrial integrity of NSCs treated with cisplatin.

\section{Effect of Latrunculin B on transfer of mitochondria and NSC survival}

Mesenchymal stem cells (MSCs) can transfer mitochondria to other cells via formation of tunneling nanotubes
(TNTs) and protrusions $[18,40]$. To determine whether mitochondrial donation is crucial for NSC survival, we pre-incubated MSCs with latrunculin B (LatB, an inhibitor of f-actin polymerization) which has been shown to inhibit TNT formation [34].We assessed the effect of LatB on mitochondrial transfer and NSC survival in the co-cultures with MSCs labeled with mito-mcherry. LatB reduced the transfer of mito-mcherry-labeled mitochondria to cisplatin-treated NSCs (Fig. 6d and e). Interestingly, LatB did not affect transfer of mitochondria to untreated NSCs (Figs. 6b and e). Moreover, LatB-treated MSCs were no longer able to promote survival of cisplatin-treated NSCs (Fig. 6f) indicating that mitochondrial transfer by MSC contributes to NSC survival.

\section{Overexpression of Miro1 in MSC increases mitochondrial transfer and promotes NSC survival}

Miro-1 is an essential mediator of microtubule-based mitochondrial motility and contributes to mitochondrial transfer between cells [2]. In our search to improve mitochondrial 


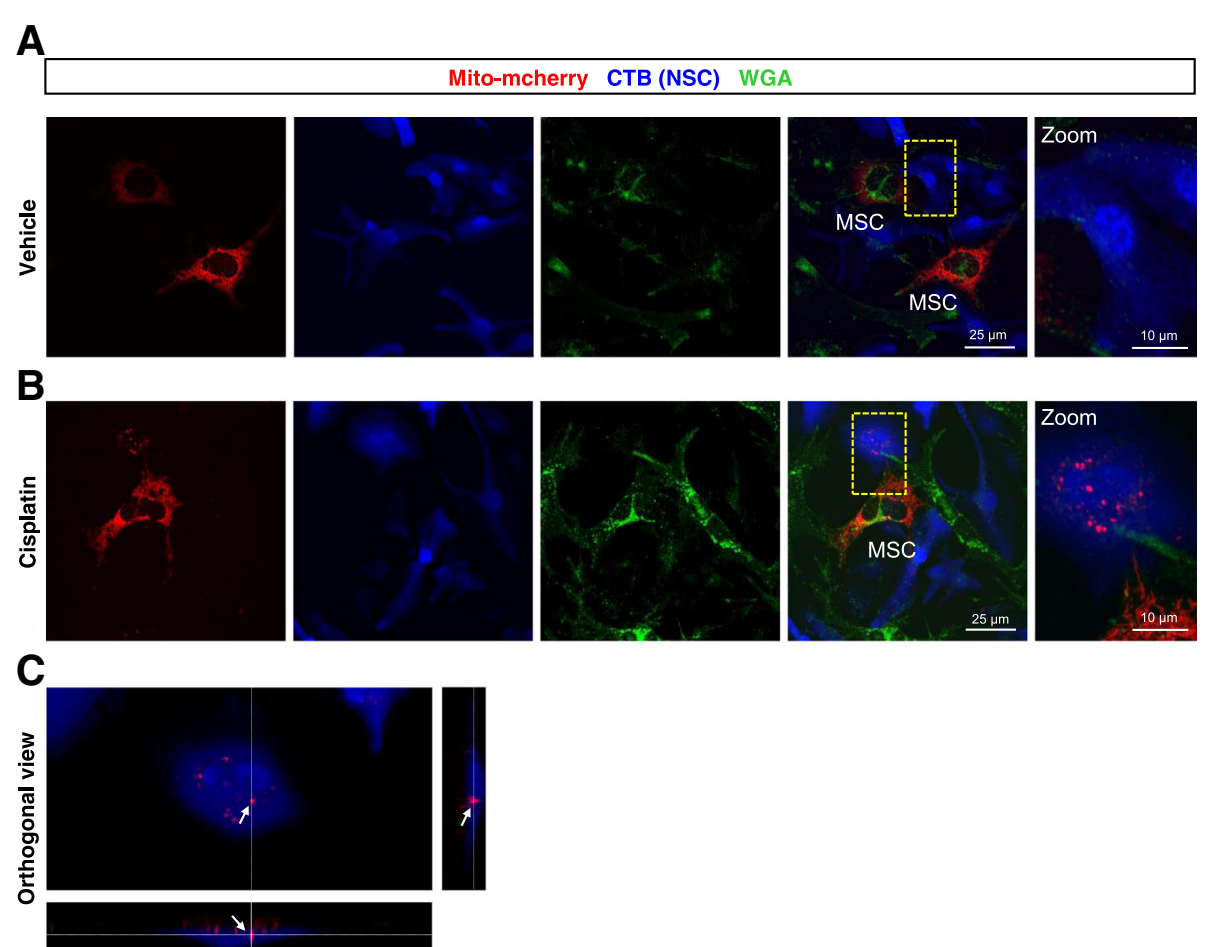

Fig. 4 Representative confocal images of Neuronal stem cells (NSCs) and mesenchymal stem cells (MSCs) co-cultures. NSCs were treated with either $1 \mu \mathrm{M}$ cisplatin or vehicle for $8 \mathrm{~h}$, stained with cell tracker blue (CTB) and co-cultured with MSCs transfected with mito-mcherry plasmid (a and $\mathbf{b})$ to label the mitochondria. Co-cultures were stained with wheat germ agglutinin (WGA) AF 488 ( $\mathbf{a}$ and $\mathbf{b}$ ) to reveal cell membranes before confocal imaging. mcherry-positive mitochondria were observed in NSCs treated with cisplatin (b, right panel) showing mitochondrial transfer. (c) Orthogonal slice view of NSC containing mcherry-positive mitochondria derived from MSC

donation by MSCs, we overexpressed Miro1 in MSCs using a mitochondrial Rho GTPase 1 (Miro1)-GFP plasmid. NSCs were treated either with $1 \mu \mathrm{M}$ cisplatin or vehicle for $8 \mathrm{~h}$, and subsequently co-cultured with or without MSCs overexpressing Miro1 and mito-mcherry or MSCs transfected with control vector and mito-mcherry for $17 \mathrm{~h}$. Overexpression of Miro1 in MSCs increased mitochondrial transfer to NSCs (Figs. 7b, d and e). Moreover, overexpression of Miro1 in MSCs increased their positive effect on survival of cisplatin-treated NSCs (Fig. 7f).

\section{Discussion}

Here we show for the first time that MSCs donate mitochondria to NSCs when damaged by cisplatin in vitro. We also show for the first time that the loss of DCX+ neuronal precursors caused by administration of 2 cycles of cisplatin can be rescued by intranasal administration of MSCs. Transfer of mitochondria from MSCs to NSCs reversed the decrease in mitochondrial membrane potential of the cisplatin-treated NSCs and favored their survival after cisplatin treatment. Blocking MSC-derived mitochondrial transfer by inhibiting actin polymerization eliminated the beneficial effect of MSCs on survival of cisplatin-treated NSCs. Conversely, when mitochondrial transfer was enhanced by overexpression of the Rho-GTPase 1 (Miro1), a mitochondrial motor protein, NSC survival after cisplatin treatment further increased. Collectively, our data support the model that MSCs can transfer mitochondria to damaged NSCs via formation of protrusions and tubular structures to rescue the latter ones after cisplatin treatment.

MSCs have the potential to repair injuries by a variety of mechanisms that range from secretion of paracrine factors and transfer of proteins and RNA to the transfer of organelles such as mitochondria. It is now well recognized that MSCs can transfer mitochondria to various cell types, including epithelial cells, macrophages, cardiomyocytes, neural cells and endothelial cells, in highly toxic or cell-damaging conditions $[2,5,18,26,27,44,46]$. As mentioned in the introduction, the transfer of mitochondria has also been shown to occur in vivo and may assist in rescuing functional and bioenergetic properties of recipient cells in metabolic need [2, 5, 18, 26, 27, 44, 46].

In our in vitro model of cisplatin-induced neurotoxicity, we observed that cisplatin treatment markedly increased the number of NSCs that received mitochondria from MSCs. This finding implies that cisplatin-treated NSCs express a "danger signal" that prompts MSCs to initiate mitochondrial transfer and therefore promote reparative functions. Several danger signals have been hypothesized to function as inducers of mitochondrial 

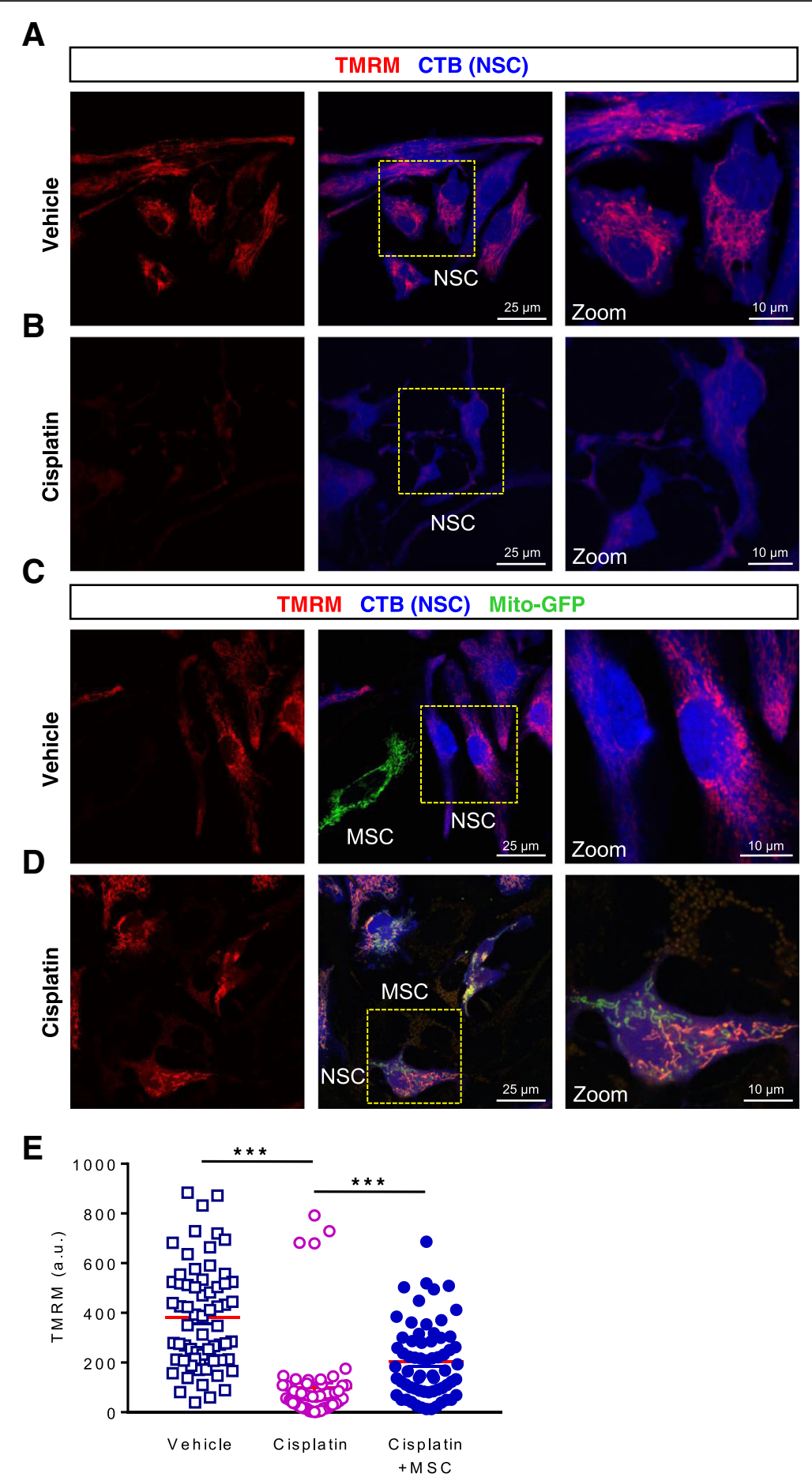

Fig. 5 Mitochondrial transfer from MSCs to NSCs damaged by cisplatin restores mitochondrial membrane potential. Neuronal stem cells (NSCs) were treated with vehicle or $1 \mu \mathrm{M}$ cisplatin for $8 \mathrm{~h}$, stained with cell tracker blue (CTB), and subsequently co-cultured for $17 \mathrm{~h}$ with or without mesenchymal stem cells (MSCs) transfected with mito-GFP to label the mitochondria. Co-cultures were stained with tetramethylrhodamine methyl ester (TMRM) and imaged. Confocal images of co-cultures show that NSCs exhibited a bright TMRM signal in control conditions (a and $\mathbf{c}$ ) that was markedly reduced after cisplatin treatment (b). The mitochondrial membrane potential in cisplatin-treated NSCs was restored after receiving MSC-derived mitochondria labeled in green (d). MSCs do not uptake TMRM as well as NSCs (Additional file 1: Figure S1). TMRM fluorescence was quantified in individual NSCS (e). $N=65$ cells were quantified in each group. Data are represented as means \pm SEM and were analyzed using One-way ANOVA followed by Dunn's multiple comparisons test. ${ }^{* *} P \leq 0.001$ 


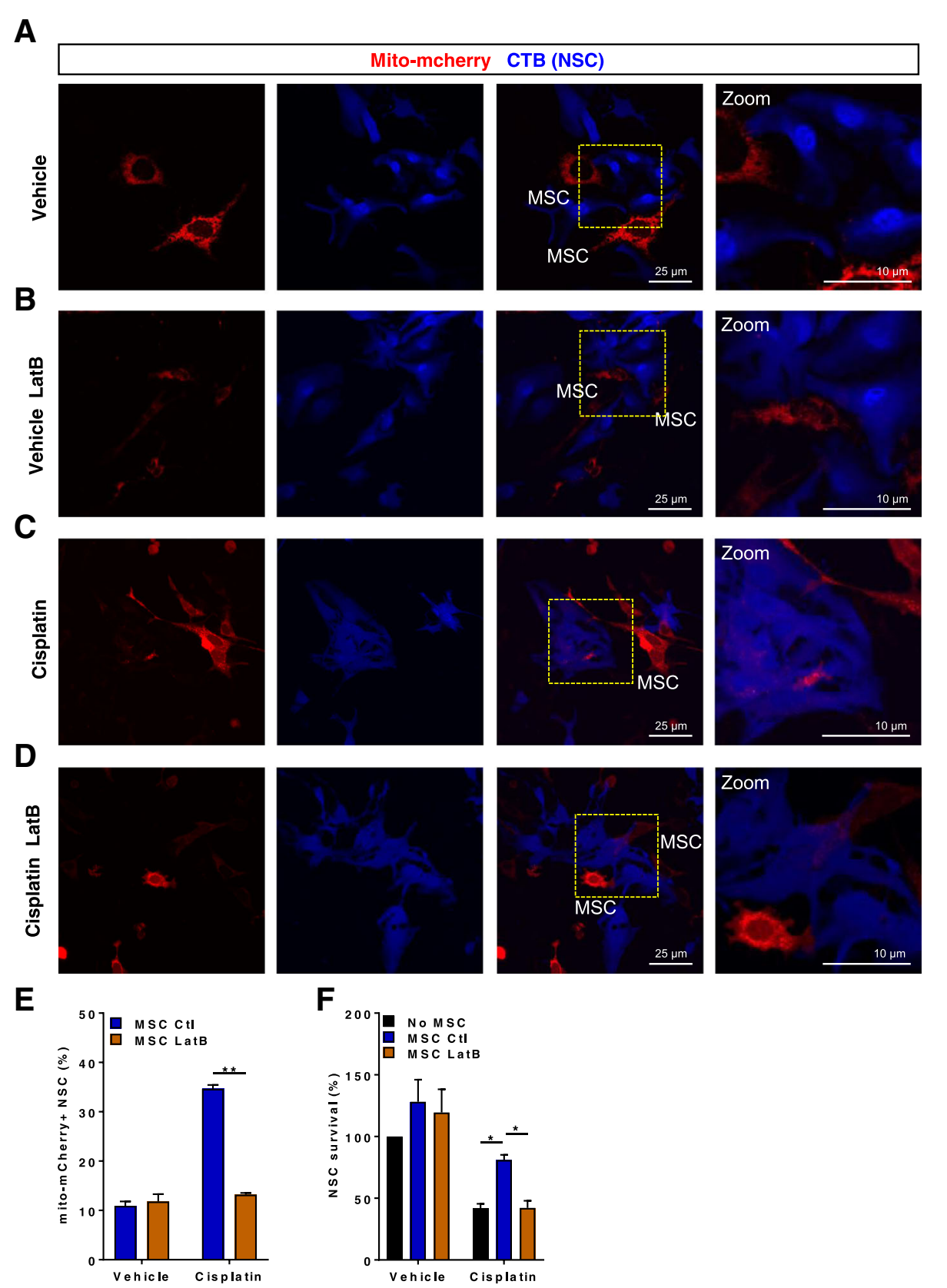

Fig. 6 Effect of Latrunculin B on transfer of mitochondria and NSC survival. Representative confocal images of Neuronal stem cells (NSCs) stained with cell tracker blue (CTB) and subsequently co-cultured for $17 \mathrm{~h}$ with mesenchymal stem cells (MSC) transfected with mito-mcherry (a-d) to label the MSC-derived mitochondria. Neuronal stem cells (NSCs) were treated with $1 \mu \mathrm{M}$ cisplatin or medium for $8 \mathrm{~h}$ followed by co-culture with mesenchymal stem cells (MSCs) treated with $2 \mu \mathrm{m}$ Latrunculin B (MSC LatB, $\mathbf{b}$ and $\mathbf{d}$ ), control MSC (MSC Ctl, a and c), or without MSC for $17 \mathrm{~h}$. Mitochondrial transfer (e) and NSC survival (f) were quantified as in Figs. 1 and 3. Data are represented as means \pm SEM of 4 independent experiments and were analyzed using two-way ANOVA followed by Bonferroni's post-hoc test. ${ }^{* *} P<0.01$; $P<0.05$

transfer. One possibility is that cells in need release damaged mitochondria and mtDNA that can then be recognized by MSCs via receptors for damage-associated molecular patterns, including toll-like receptors [12]. The actual uptake of damaged mitochondria by MSCs was shown to be crucial for activating MSCs to rescue damaged cardiomyocytes or human umbilical-vein endothelial cells, both in vitro and in vivo [29].

Intercellular communication is crucial for the development and maintenance of tissue growth, differentiation, and regeneration. Cells are capable of establishing direct contact through various types of cell connections, such 


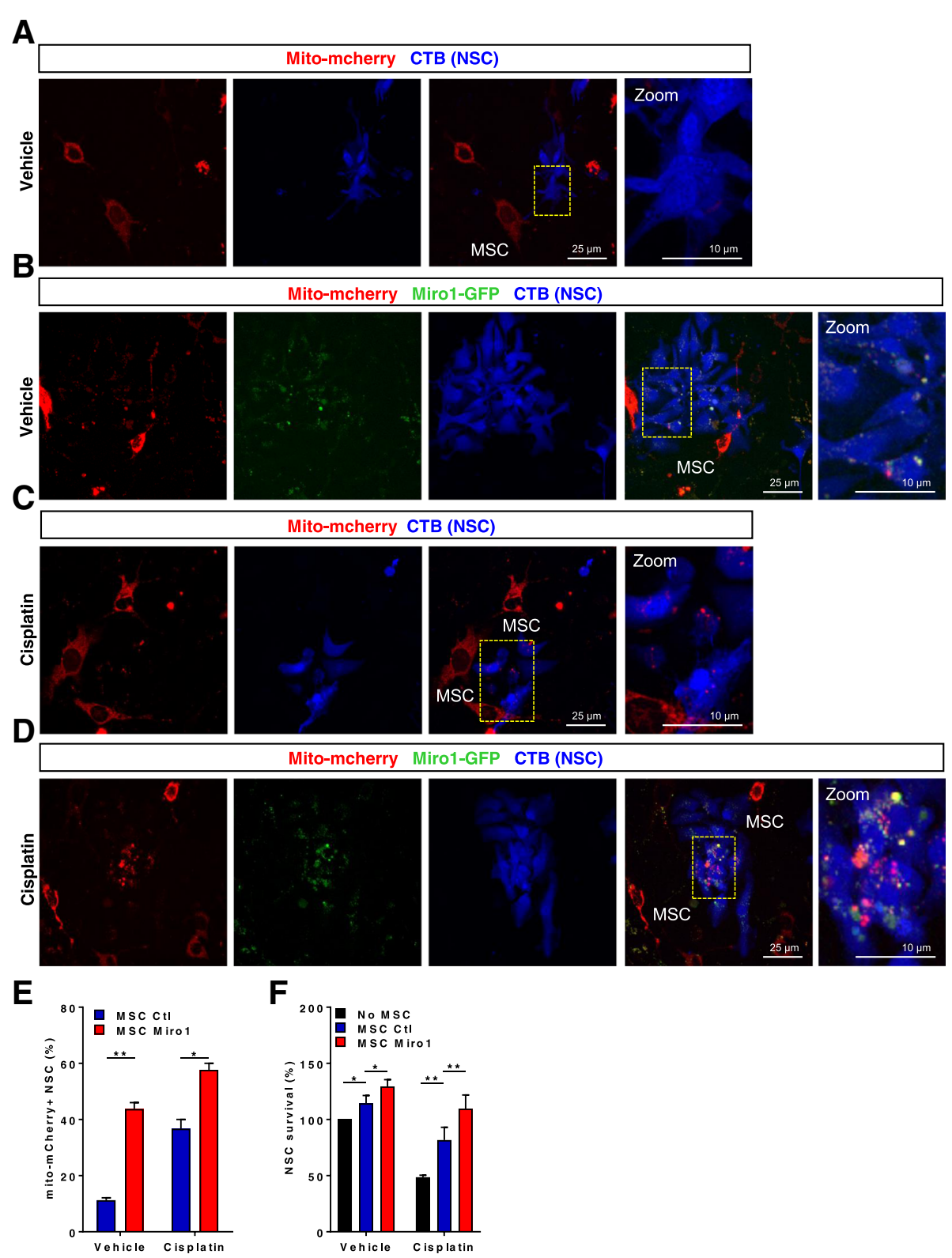

Fig. 7 Overexpression of Miro1 in MSCs boosts NSC survival and enhances mitochondrial transfer to injured NSCs. Representative confocal images of Neuronal stem cells (NSCs) stained with cell tracker blue (CTB) and subsequently co-cultured for $17 \mathrm{~h}$ with mesenchymal stem cells (MSC) transfected with mito-mcherry (a-d) and miro1-GFP (b, d) to label the MSC-derived mitochondria. Neuronal stem cells (NSCs) were treated with $1 \mu \mathrm{M}$ cisplatin for $8 \mathrm{~h}$ and then co-cultured for $17 \mathrm{~h}$ with mesenchymal stem cells (MSCs) overexpressing Miro1 GTPase (MSC Miro 1), MSCs transfected with empty vector (MSC Ctl), or without MSCs. Mitochondrial transfer (e) and survival (f) were assessed as in Fig. 6. Data were analyzed by two-way ANOVA followed by Bonferroni's post-hoc test. ${ }^{*} P<0.01$; ${ }^{*} P<0.05$

as formation of cytoplasmic TNTs that enable the transfer of organelles such as mitochondria from one cell to another. Studies have reported that one of the means by which MSCs make contact with injured cells and transfer their mitochondria is via formation of these TNTs $[2,18,26,27]$. Our finding that LatB reduces mitochondrial transfer indicates that MSCs use TNTs for delivering their mitochondria. The molecular signal inducing the formation of TNTs is still unclear and seems to differ between cell types [40]. Studies conducted in immune cells and HEK293T cells highlight the involvement of the M-Sec pathway, a 73-kDa cytosolic protein also known as tumor necrosis factor $\alpha$-induced protein 2 or B94, in inducing the membrane protrusion that is one of the first steps in the formation of TNTs $[14,32]$. In addition, the tumor suppressor molecule p53 and the Akt/PI3K/mTOR signaling pathway have been shown to play a role in TNT formation in astrocytes [42]. 
Wang et al. [42] also found that p53 activation is crucial for TNT formation since genetic ablation of p53 prevented formation of TNTs in rat hippocampal co-cultures of astrocytes and neurons. In line with these findings, we have preliminary data indicating that prevention of mitochondrial accumulation of p53 by the mitochondrial protectant pifithrin- $\mu$, decreased the transfer of mitochondria to damaged NSCs in vitro (data not shown). Furthermore, we recently showed that in vivo, cisplatin treatment rapidly induced translocation of p53 to mitochondria in the brain. Inhibition of p53 translocation to mitochondria prevented cisplatin-induced mitochondrial dysfunction of neuronal synaptosomes [7]. Therefore, we propose that translocation of p53 to the mitochondria of the damaged acceptor cell is part of the complex that confers a danger signal to the donor cell and prompt MSCs to transfer healthy mitochondria to NSCs.

Mitochondrial transfer between donor and acceptor cells requires mitochondrial movement along the actin cytoskeleton of the cells. This movement of mitochondria is regulated by the mitochondrial membrane Rho-GTPase 1 (Miro1), which binds the cytoplasmic adaptor protein milton and kinesin heavy chain through its cytoplasmic domains, thereby connecting mitochondria to the actin cytoskeleton. We show that Miro1 overexpression in MSCs increased the transfer of mitochondria and enhanced the rescue potential of MSCs when co-cultured with cisplatin-damaged NSCs. Ahmad et al. [2] demonstrated in an in vitro model using lung epithelial cells that genetic manipulation of MSCs to overexpress Miro1 resulted in higher efficiency of transfer from MSCs to epithelial cells. Interestingly, intravenous administration of MSCs overexpressing Miro1 improved the efficacy of MSCs to reduce rotenone-induced lung injury [2]. The mechanism of action of Miro1 is complex but Schuler et al. recently showed using mouse embryonic fibroblasts that increasing Miro1 in MSCs may lead to better positioning of mitochondria at the leading edge of the cytoplasm instead of in the perinuclear area, allowing higher cytoplasmic energy redistribution and thereby favoring protrusion formation and MSC migration [36].

We do not yet know how mitochondria of donor origin rescue mitochondrial health and promote survival of the damaged acceptor NSCs. One possibility is that the donor mitochondria fuse with mitochondria in the acceptor cell, thereby restoring bioenergetic efficiency. Another potential mechanism is that the acceptor cell discards the damaged mitochondria and instead hijacks the donated mitochondria for energy production. Future research is needed to investigate how the MSC-derived mitochondria communicate with the acceptor cell cellular machinery to foster cellular health.

MSCs are becoming excellent candidates for regenerative strategies to restore brain damage after traumatic brain injury, stroke, hypoxia-ischemia, and neurodegenerative disorders like Alzheimer disease [9-11, 24, 25, 45]. We recently observed that nasal administration of MSCs can restore the cognitive deficits that arise after cisplatin treatment $[6,16]$. In relation to this finding, it is of interest that we observed that cisplatin-induced loss of DCX+ neuroblasts was reversed by intranasal administration of MSCs as well. We propose that MSC-mediated transfer of mitochondria to NSCs reverses the neurotoxic effects of cisplatin, thereby facilitating cognitive processes.

The improvement in mitochondrial integrity and survival of NSCs in the presence of MSCs is likely not limited to the transfer of mitochondria alone. MSCs are known to favorably influence the growth factor milieu, which could also aid in rescuing cisplatin-damaged NSCs [38]. However, when we blocked the transfer of mitochondria to NSCs using LatB, which depolymerizes F-actin and thereby inhibits TNT formation, we no longer observed the positive effect of MSCs on the NSC survival in vitro. These findings indicate that mitochondrial transfer plays a major role in the rescue of NSCs damaged by cisplatin.

In conclusion, we propose that the transfer of healthy mitochondria is an important mechanism underlying the regenerative effects of MSCs in the brain.

\section{Additional file}

Additional file 1: Figure S1. Representative images of MSC harboring GFP labeled mitochondria and TMRM staining. MSCs were transfected with mito-GFP, stained with TMRM and imaged. MSCs do not exhibit a strong TMRM signal as compared to NSCs. (PDF $106 \mathrm{~kb}$ )

\section{Abbreviations}

ATP: Adenosine triphosphate; CTB: Cell Tracker Blue fluorescent probe; DCX: Doublecortin; DG: Dentate Gyrus; FCCP: Carbonilcyanide ptriflouromethoxyphenylhydrazone; GFAP: Glial fibrillary acidic protein; GFP: Green fluorescent protein; LatB: Latrunculin B; Miro1: Mitochondrial Rho GTPase 1; MSC: Mesenchymal stem cell; NSC: Neuronal stem cell; SVZ: Subventricular Zone; TMRM: Tetramethylrhodamine methyl ester; TNT: Tunneling nanotube; WGA: Wheat germ agglutinin

\section{Acknowledgments}

We are greatly indebted to Dr. Manon Benders for her constructive advice during this study. The authors also acknowledge Jeanie F. Woodruff, BS, ELS, for scientific editing.

\section{Funding}

This work was supported by the National Institutes of Health grants R01CA208371, R01CA230512 and R01CA227064 to Cobi J. Heijnen and Annemieke Kavelaars. The National Institutes of Health played no role in the study design; in the collection, analysis and interpretation of data; in the writing of the report; or in the decision to submit the article for publication.

Availability of data and materials

All data generated or analyzed during this study are included in this published article [and its supplementary information files]. 


\section{Authors' contributions}

$N B, A K$ and $C J H$ designed the study. NB, GC. performed the experiments. NB, $\mathrm{GC}$, AK and $\mathrm{CJH}$ interpreted the data. $\mathrm{NB}, \mathrm{AK}$ and $\mathrm{CJH}$ wrote the manuscript. All authors read and approved the final version.

\section{Ethics approval and consent to participate}

All animal procedures were in accordance with the National Institute of Health Guidelines for the Care and Use of Laboratory Animals and were approved by the Institutional Animal Care and Use Committee (IACUC) of M.D. Anderson Cancer Center.

\section{Consent for publication}

Not applicable.

\section{Competing interests}

None.

\section{Publisher's Note}

Springer Nature remains neutral with regard to jurisdictional claims in published maps and institutional affiliations.

\section{Received: 12 October 2018 Accepted: 2 December 2018} Published online: 12 December 2018

\section{References}

1. Ahles TA, Root JC, Ryan EL (2012) Cancer- and cancer treatment-associated cognitive change: an update on the state of the science. J Clin Oncol 30: 3675-3686. https://doi.org/10.1200/JCO.2012.43.0116

2. Ahmad T, Mukherjee S, Pattnaik B, Kumar M, Singh S, Kumar M, Rehman R, Tiwari BK, Jha KA, Barhanpurkar AP et al (2014) Miro1 regulates intercellular mitochondrial transport \& enhances mesenchymal stem cell rescue efficacy. EMBO J 33:994-1010. https://doi.org/10.1002/embj.201386030

3. Andres AL, Gong X, Di K, Bota DA (2014) Low-doses of cisplatin injure hippocampal synapses: a mechanism for 'chemo' brain? Exp Neurol 255: 137-144. https://doi.org/10.1016/j.expneurol.2014.02.020

4. Baameur F, Singhmar P, Zhou Y, Hancock JF, Cheng X, Heijnen CJ, Kavelaars A (2016) Epac1 interacts with importin beta1 and controls neurite outgrowth independently of cAMP and Rap1. Sci Rep 6:36370. https://doi. org/10.1038/srep36370

5. Babenko VA, Silachev DN, Zorova LD, Pevzner IB, Khutornenko AA, Plotnikov EY, Sukhikh GT, Zorov DB (2015) Improving the post-stroke therapeutic potency of mesenchymal multipotent stromal cells by Cocultivation with cortical neurons: the role of crosstalk between cells. Stem Cells Transl Med 4:1011-1020. https://doi.org/10.5966/sctm.2015-0010

6. Chiu GS, Boukelmoune N, Chiang ACA, Peng B, Rao V, Kingsley C, Liu HL, Kavelaars A, Kesler SR, Heijnen CJ (2018) Nasal administration of mesenchymal stem cells restores cisplatin-induced cognitive impairment and brain damage in mice. Oncotarget 9:35581-35597. https://doi.org/10. 18632/oncotarget.26272

7. Chiu GS, Maj MA, Rizvi S, Dantzer R, Vichaya EG, Laumet G, Kavelaars A, Heijnen CJ (2017) Pifithrin-mu prevents cisplatin-induced Chemobrain by preserving neuronal mitochondrial function. Cancer Res 77:742-752. https:// doi.org/10.1158/0008-5472.CAN-16-1817

8. Danielyan L, Beer-Hammer S, Stolzing A, Schafer R, Siegel G, Fabian C, Kahle P, Biedermann T, Lourhmati A, Buadze M et al (2014) Intranasal delivery of bone marrow-derived mesenchymal stem cells, macrophages, and microglia to the brain in mouse models of Alzheimer's and Parkinson's disease. Cell Transplant 23(Suppl 1):S123-S139. https://doi.org/10.3727/ 096368914 X684970

9. Donega V, Nijboer CH, Braccioli L, Slaper-Cortenbach I, Kavelaars A, van Bel F, Heijnen CJ (2014) Intranasal administration of human MSC for ischemic brain injury in the mouse: in vitro and in vivo neuroregenerative functions. PLoS One 9:e112339. https://doi.org/10.1371/journal.pone.0112339

10. Donega V, Nijboer CH, van Tilborg G, Dijkhuizen RM, Kavelaars A, Heijnen CJ (2014) Intranasally administered mesenchymal stem cells promote a regenerative niche for repair of neonatal ischemic brain injury. Exp Neurol 261:53-64. https://doi.org/10.1016/j.expneurol.2014.06.009

11. Donega V, van Velthoven CT, Nijboer CH, van Bel F, Kas MJ, Kavelaars A, Heijnen CJ (2013) Intranasal mesenchymal stem cell treatment for neonatal brain damage: long-term cognitive and sensorimotor improvement. PLoS One 8:e51253. https://doi.org/10.1371/journal.pone.0051253
12. Galluzzi L, Kepp O, Kroemer G (2012) Mitochondria: master regulators of danger signalling. Nat Rev Mol Cell Biol 13:780-788. https://doi.org/10.1038/ nrm3479

13. Gong X, Schwartz PH, Linskey ME, Bota DA (2011) Neural stem/progenitors and glioma stem-like cells have differential sensitivity to chemotherapy. Neurology 76:1126-1134. https://doi.org/10.1212/WNL.0b013e318212a89f

14. Hase K, Kimura S, Takatsu H, Ohmae M, Kawano S, Kitamura H, Ito M, Watarai H, Hazelett CC, Yeaman C et al (2009) M-sec promotes membrane nanotube formation by interacting with Ral and the exocyst complex. Nat Cell Biol 11:1427-1432. https://doi.org/10.1038/ncb1990

15. Hayakawa K, Esposito E, Wang X, Terasaki Y, Liu Y, Xing C, Ji X, Lo EH (2016) Transfer of mitochondria from astrocytes to neurons after stroke. Nature 535:551-555. https://doi.org/10.1038/nature18928

16. Huo X, Reyes TM, Heijnen CJ, Kavelaars A (2018) Cisplatin treatment induces attention deficits and impairs synaptic integrity in the prefrontal cortex in mice. Sci Rep 8:17400. https://doi.org/10.1038/s41598-018-35919-x

17. Islam MN, Das SR, Emin MT, Wei M, Sun L, Westphalen K, Rowlands DJ, Quadri SK, Bhattacharya S, Bhattacharya J (2012) Mitochondrial transfer from bone-marrow-derived stromal cells to pulmonary alveoli protects against acute lung injury. Nat Med 18:759-765. https://doi.org/10.1038/nm.2736

18. Jackson MV, Morrison TJ, Doherty DF, McAuley DF, Matthay MA, Kissenpfennig A, O'Kane CM, Krasnodembskaya AD (2016) Mitochondrial transfer via tunneling nanotubes is an important mechanism by which mesenchymal stem cells enhance macrophage phagocytosis in the in vitro and in vivo models of ARDS. Stem Cells 34:2210-2223. https://doi.org/10. 1002/stem.2372

19. Kelland $L$ (2007) The resurgence of platinum-based cancer chemotherapy. Nat Rev Cancer 7:573-584. https://doi.org/10.1038/nrc2167

20. Kesler S, Janelsins M, Koovakkattu D, Palesh O, Mustian K, Morrow G, Dhabhar FS (2013) Reduced hippocampal volume and verbal memory performance associated with interleukin- 6 and tumor necrosis factor-alpha levels in chemotherapy-treated breast cancer survivors. Brain Behav Immun 30(Suppl):S109-S116. https://doi.org/10.1016/j.bbi.2012.05.017

21. Kesler SR, Blayney DW (2016) Neurotoxic effects of anthracycline- vs nonanthracycline-based chemotherapy on cognition in breast Cancer survivors. JAMA Oncol 2:185-192. https://doi.org/10.1001/jamaoncol.2015. 4333

22. Kesler SR, Watson CL, Blayney DW (2015) Brain network alterations and vulnerability to simulated neurodegeneration in breast cancer. Neurobiol Aging 36:2429-2442. https://doi.org/10.1016/j.neurobiolaging.2015.04.015

23. Krukowski K, Ma J, Golonzhka O, Laumet GO, Gutti T, van Duzer JH, Mazitschek R, Jarpe MB, Heijnen CJ, Kavelaars A (2017) HDAC6 inhibition effectively reverses chemotherapy-induced peripheral neuropathy. Pain 158: 1126-1137. https://doi.org/10.1097/j.pain.0000000000000893

24. Lee HJ, Lee JK, Lee H, Carter JE, Chang JW, Oh W, Yang YS, Suh JG, Lee BH, Jin HK et al (2012) Human umbilical cord blood-derived mesenchymal Stem Cells improve neuropathology and cognitive impairment in an Alzheimer's disease mouse model through modulation of neuroinflammation. Neurobiol Aging 33:588-602. https://doi.org/10.1016/j.neurobiolaging.2010.03.024

25. Lee JK, Jin HK, Bae JS (2009) Bone marrow-derived mesenchymal stem cells reduce brain amyloid-beta deposition and accelerate the activation of microglia in an acutely induced Alzheimer's disease mouse model. Neurosci Lett 450:136-141. https://doi.org/10.1016/..neulet.2008.11.059

26. Li X, Zhang Y, Yeung SC, Liang Y, Liang X, Ding Y, Ip MS, Tse HF, Mak JC, Lian Q (2014) Mitochondrial transfer of induced pluripotent stem cellderived mesenchymal stem cells to airway epithelial cells attenuates cigarette smoke-induced damage. Am J Respir Cell Mol Biol 51:455-465. https://doi.org/10.1165/rcmb.2013-05290C

27. Liu K, Ji K, Guo L, Wu W, Lu H, Shan P, Yan C (2014) Mesenchymal stem cells rescue injured endothelial cells in an in vitro ischemia-reperfusion model via tunneling nanotube like structure-mediated mitochondrial transfer. Microvasc Res 92:10-18. https://doi.org/10.1016/j.mvr.2014.01.008

28. Lomeli N, Di K, Czerniawski J, Guzowski JF, Bota DA (2017) Cisplatin-induced mitochondrial dysfunction is associated with impaired cognitive function in rats. Free Radic Biol Med 102:274-286. https://doi.org/10.1016/j. freeradbiomed.2016.11.046

29. Mahrouf-Yorgov M, Augeul L, Da Silva CC, Jourdan M, Rigolet M, Manin S, Ferrera R, Ovize M, Henry A, Guguin A et al (2017) Mesenchymal Stem Cells sense mitochondria released from damaged cells as danger signals to activate their rescue properties. Cell Death Differ 24:1224-1238. https://doi. org/10.1038/cdd.2017.51 
30. Maj MA, Ma J, Krukowski KN, Kavelaars A, Heijnen CJ (2017) Inhibition of mitochondrial p53 accumulation by PFT-mu prevents cisplatin-induced peripheral neuropathy. Front Mol Neurosci 10:108. https://doi.org/10.3389/ fnmol.2017.00108

31. McCloy RA, Rogers S, Caldon CE, Lorca T, Castro A, Burgess A (2014) Partial inhibition of $\mathrm{Cdk} 1$ in $\mathrm{G} 2$ phase overrides the $\mathrm{SAC}$ and decouples mitotic events. Cell Cycle 13:1400-1412. https://doi.org/10.4161/cc.28401

32. Ohno H, Hase K, Kimura S (2010) M-sec: emerging secrets of tunneling nanotube formation. Commun Integr Biol 3:231-233

33. Rego AC, Vesce S, Nicholls DG (2001) The mechanism of mitochondrial membrane potential retention following release of cytochrome $\mathrm{c}$ in apoptotic GT1-7 neural cells. Cell Death Differ 8:995-1003. https://doi.org/ 10.1038/sj.cdd.4400916

34. Rustom A, Saffrich R, Markovic I, Walther P, Gerdes HH (2004) Nanotubular highways for intercellular organelle transport. Science 303:1007-1010. https://doi.org/10.1126/science.1093133

35. Sancak Y, Markhard AL, Kitami T, Kovacs-Bogdan E, Kamer KJ, Udeshi ND, Carr SA, Chaudhuri D, Clapham DE, Li AA et al (2013) EMRE is an essential component of the mitochondrial calcium uniporter complex. Science 342: 1379-1382. https://doi.org/10.1126/science.1242993

36. Schuler MH, Lewandowska A, Caprio GD, Skillern W, Upadhyayula S, Kirchhausen T, Shaw JM, Cunniff B (2017) Miro1-mediated mitochondrial positioning shapes intracellular energy gradients required for cell migration. Mol Biol Cell 28:2159-2169. https://doi.org/10.1091/mbc.E16-10-0741

37. Seigers R, Fardell JE (2011) Neurobiological basis of chemotherapy-induced cognitive impairment: a review of rodent research. Neurosci Biobehav Rev 35:729-741. https://doi.org/10.1016/j.neubiorev.2010.09.006

38. van Velthoven CT, Kavelaars A, van Bel F, Heijnen CJ (2010) Repeated mesenchymal stem cell treatment after neonatal hypoxia-ischemia has distinct effects on formation and maturation of new neurons and oligodendrocytes leading to restoration of damage, corticospinal motor tract activity. and sensorimotor function J Neurosci 30:9603-9611. https:// doi.org/10.1523/JNEUROSCI.1835-10.2010

39. Vichaya EG, Chiu GS, Krukowski K, Lacourt TE, Kavelaars A, Dantzer R, Heijnen CJ, Walker AK (2015) Mechanisms of chemotherapy-induced behavioral toxicities. Front Neurosci 9:131. https://doi.org/10.3389/fnins.2015.00131

40. Vignais ML, Caicedo A, Brondello JM, Jorgensen C (2017) Cell connections by tunneling nanotubes: effects of mitochondrial trafficking on target cell metabolism, homeostasis, and response to therapy. Stem Cells Int 2017: 6917941. https://doi.org/10.1155/2017/6917941

41. Villeda SA, Luo J, Mosher Kl, Zou B, Britschgi M, Bieri G, Stan TM, Fainberg N, Ding Z, Eggel A et al (2011) The ageing systemic milieu negatively regulates neurogenesis and cognitive function. Nature 477:90-94. https://doi.org/10. 1038/nature10357

42. Wang $Y$, Cui J, Sun $X$, Zhang $Y$ (2011) Tunneling-nanotube development in astrocytes depends on p53 activation. Cell Death Differ 18:732-742. https:// doi.org/10.1038/cdd.2010.147

43. Wefel JS, Schagen SB (2012) Chemotherapy-related cognitive dysfunction. Curr Neurol Neurosci Rep 12:267-275. https:/doi.org/10.1007/s11910-012-0264-9

44. Yao Y, Fan XL, Jiang D, Zhang Y, Li X, Xu ZB, Fang SB, Chiu S, Tse HF, Lian Q et al (2018) Connexin 43-Mediated Mitochondrial Transfer of iPSC-MSCS Alleviates Asthma Inflammation. Stem Cell Reports 11:1120-1135. https:// doi.org/10.1016/j.stemcr.2018.09.012

45. Zhang R, Liu Y, Yan K, Chen L, Chen XR, Li P, Chen FF, Jiang XD (2013) Antiinflammatory and immunomodulatory mechanisms of mesenchymal stem cell transplantation in experimental traumatic brain injury. $J$ Neuroinflammation 10:106. https://doi.org/10.1186/1742-2094-10-106

46. Zhang Y, Yu Z, Jiang D, Liang X, Liao S, Zhang Z, Yue W, Li X, Chiu SM, Chai YH et al (2016) iPSC-MSCs with High Intrinsic MIRO1 and Sensitivity to TNFalpha Yield Efficacious Mitochondrial Transfer to Rescue AnthracyclineInduced Cardiomyopathy. Stem Cell Reports 7:749-763. https://doi.org/10. 1016/j.stemcr.2016.08.009

47. Zhao C, Deng W, Gage FH (2008) Mechanisms and functional implications of adult neurogenesis. Cell 132:645-660. https://doi.org/10.1016/j.cell.2008. 01.033

\section{Ready to submit your research? Choose BMC and benefit from:}

- fast, convenient online submission

- thorough peer review by experienced researchers in your field

- rapid publication on acceptance

- support for research data, including large and complex data types

- gold Open Access which fosters wider collaboration and increased citations

- maximum visibility for your research: over $100 \mathrm{M}$ website views per year

At BMC, research is always in progress.

Learn more biomedcentral.com/submissions 